\title{
Efficacy and Safety of Endoscopic Resection and Open Surgery for Treating Thyroid Diseases: A Meta-Analysis
}

\author{
JIANFENG WENG, HUAZHOU ZHAO, KEYING CUI AND JIANMIAO HE*
}

Department of General Surgery, Eighth Medical Center of General Hospital of the People's Liberation Army, Beijing 100091, China

Weng et al.: Treatment of Thyroid Diseases

\begin{abstract}
To explore the efficacy and safety of endoscopic resection and open surgery for treating thyroid diseases using meta-analysis. Related data in computer retrieval of Cochrane Library, American National Library of Medicine Biomedical Information Retrieval System (Pub Med), Dutch Medicine Abstracts (Embase), China Biomedical Literature Database (CBM), China Academic Journals Full-text Database (CNKI), Chinese Science and Technology Journal Database (VIP), and Western Language Biomedical Journal Literature Database (EMC) from December 2019 to April 2020 were retrieved. Relevant journals and included references were manually searched. Randomized controlled trials regarding the effects of endoscopic thyroid surgery and open thyroid surgery on the immune response of thyroid diseases were included. Two reviewers conducted independent data extraction, quality evaluation and cross-checking according to inclusion and exclusion criteria. RevMan 5.1.4 software was employed. Eight non-randomized controlled retrospective cohort studies were finally included. The curative rate $(168 / 185,90.81 \%$ vs. $194 / 383,50.65 \%, p<0.01$ ) was significantly higher in endoscopic resection group and the difference between local recurrence rates $(2 / 366,0.55 \%$ vs. $83 / 603,13.76 \%, p<0.01)$ was significant. There was no significant difference between bleeding rate $(1 / 325,0.31 \%$ vs. $4 / 407,0.98 \%, p=0.41)$. In terms of performing thyroid surgery under endoscopy, the efficacy of adjuvant treatment under endoscopy is significantly better than that of control group and the safety is similar to that of control group. Endoscopic treatment is suitable for treating thyroid diseases. However, the conclusions still need to be verified by multicenter randomized controlled trials with large sample sizes.
\end{abstract}

Key words: Endoscopy, thyroid, hemostasis, hypoparathyroidism

Thyroid diseases are more common in female patients. The traditional surgical incision is located just in front of the exposed neck, which affects the appearance, especially for female patients, because this shortcoming often affects them to choose correct and timely surgical treatment. Endoscopic thyroid surgery is a relatively new field. The reason why it has not been widely carried out is that the neck space is narrow and a surgical operation space must be created; the thyroid is a blood-rich organ and surgery is prone to bleeding. The equipment requirements are high. With the advancement of surgical technology, such as the establishment of various effective methods to expand the surgical space, especially the application of new equipment, the ultrasonic knife has promoted the rapid development of endoscopic thyroid surgery. However, the subjects included in the study were not for Lateral spreading tumors (LST) patients. At present, there are no guidelines for the treatment of LST at home and abroad and the effectiveness and safety of Endoscopic submucosal dissection (ESD) and Endoscopic mucosal resection (EMR) resection of colorectal LST need to be further evaluated. This systematic review aims to use the principles and methods of evidence-based medicine and strictly follow the requirements of the Cochrane systematic review to comprehensively search and screen randomized controlled trials (RCT) related to endoscopic thyroid surgery and open thyroid surgery to evaluate whether this technique is effective, feasible and safe. 


\section{METHODS}

\section{Search strategy:}

Two reviewers searched Pub Med, Cochrane Central library, Embase, China Biomedical Literature Database, CNKI and Wanfang Database by computer to find all the literature comparing ESD and EMR resection of colorectal LST. The Chinese keywords are endoscopic mucosal resection, endoscopic mucosal dissection and colorectal lateral developmental tumor. English keywords are endoscopic mucosal resection, endoscopic submucosal dissection and colorectal laterally spreading tumor

Inclusion criteria: Patients with colorectal LST diagnosed pathologically, regardless of race or nationality; the experimental group and the control group were treated with ESD and EMR for colorectal LST; the literature contains the following main observation indicators, such as tumor mass resection rate, complete resection rate, local recurrence rate, operation time, postoperative perforation rate and delayed bleeding rate.

Exclusion criteria: case report, review; only one endoscopic treatment method was reported in the study.

Literature quality evaluation: Newcastle data extraction is used for literature quality evaluation: data extraction is performed after reading the full text, which is done independently by two reviewers. In case of dispute, a third reviewer will intervene for discussion. The content includes sample size, basic data of the research object, measurement indicators and followup duration. The data related to endoscopic mucosal resection (EPMR) in the original literature were all included in the EMR group.

\section{Data processing:}

Quality evaluation and data extraction: According to the RCT quality evaluation standard of Cochrane System Evaluation Guide 5.1.4, two reviewers individually evaluate the quality of the included literature and then cross-check. If there is a disagreement, the third reviewer will assist in the resolution.

Evaluation contents: Random grouping method; assignment hiding; blind method; incomplete result data; selective reporting of results; other biases. Three reviewers independently screened the literature according to the inclusion criteria, used the pre-designed literature extraction form to extract information and cross-checked it and discussed and resolved any differences. If the information in the literature is incomplete, further contact the author of the literature research to obtain relevant information. If the relevant data is not obtained in the end, the literature will be eliminated.

\section{Surgical methods:}

Before the start of endoscopic thyroid surgery, mark the mass, thyroid cartilage nodules, midline, sternocleidomastoid muscle and incision site. Enter from the space between the deep and shallow fascia and the anterior area of the neck is on the deep surface of the platy muscles. Mastoid muscles, anterior cervical muscles and trachea are used as anatomical landmarks to operate. Make full use of the operating space. Like traditional surgery, the flap should be separated to the lower edge of the thyroid cartilage and the midline of the neck should be fully cut. Generally, an ultrasonic knife is used to separate and remove the thyroid from the lower pole and the side. Those with difficulty can transcribe the anterior cervical muscle of the affected side or use a thin thread to pull the anterior cervical muscle through the skin. You can also pull the mass with sutures. Our experience of nearly 100 cases has found that there is no need to routinely transcribe the cervical anterior muscle for cases with a mass diameter of $<5.0$ $\mathrm{cm}$, which can reduce surgical trauma and shorten the operation time, as well as reduce postoperative local adhesions; intraoperative hemostasis is necessary thorough. Benign lesions should be resected within the proper thyroid capsule as much as possible. When operating on the back of the thyroid, blunt separation should be gently pushed to reduce the frequency and time of ultrasonic knife use. If possible, ligature can be used; avoid accidental injury to the recurrent laryngeal nerve and parathyroid glands.

\section{Statistical analysis:}

Statistical package for the social sciences (SPSS) 22.0 software and RevMan 5.1.4 software were used for statistical analysis. Measurement data were analyzed by weighted mean difference (WMD) or standard mean difference (SMD) and each effect size was expressed in a $95 \%$ confidence interval (CI). According to the possible heterogeneity factors, the subgroup analysis was carried out and the $r$ value was used to test the heterogeneity among the studies. There is statistical homogeneity among the subgroups ( $\mathrm{p} \geq 0.1, \mathrm{I} 2 \leq 25 \%$ ). The fixed effects model is used to calculate the total results when there is statistical heterogeneity $(\mathrm{p}<0.1$, I $>25 \%$ ). The effect model calculates the total result. For example, only one study in the subgroup still uses the same statistics as meta-analysis. $\mathrm{p}<0.05$ indicates 
that difference is statistically significant.

According to the above mentioned search strategy, the literature search results obtained a total of 218 Chinese and English documents from the relevant database and a total of 11 documents were retrieved from related journals. Duplicate documents were eliminated and 220 documents that met the standard were initially obtained. After reading the title and abstract, 23 documents were obtained. After reading the full text, $7 \operatorname{articles}^{[1]}$ that meet the criteria were finally included, including 5 in English and 2 in Chinese. A total of 2192 cases were collected in 7 studies. The general characteristics mainly include sample size, sex ratio, age and followup time (Table 1). Three of the seven included studies reported the method of random assignment in detail ${ }^{[2]}$. Two papers were double-blinded ${ }^{[3]}$ and the rest were not described in detail.

Permanent recurrent laryngeal nerve palsy included studies reported on permanent recurrent laryngeal nerve palsy and 4 studies did not see the occurrence of this adverse event. The statistical heterogeneity between the studies is small $\left(\mathrm{I}^{2}=34 \%, \mathrm{p}=0.22\right)$, so the fixed effects model is used. There was no statistically significant difference in the incidence of permanent recurrent laryngeal nerve palsy between $\mathrm{TT}$ and $\mathrm{ST}(\mathrm{OR}=0.81$, $95 \%$ CI: 0.24 2.74, p=0.74; fig. 1-fig. 3).

Transient hypoparathyroidism included 7 studies that reported the occurrence of transient hypoparathyroidism during the follow-up period of patients. There was no statistical heterogeneity among the studies $\left(\mathrm{I}^{2}=0 \%\right.$, $\mathrm{p}=0.56$ ), so the fixed effects model is adopted. The results of Meta-analysis showed that the incidence of transient hypoparathyroidism after thyroidectomy (TT) was higher than that after sternothyroid muscle (ST) and the difference was statistically significant $(\mathrm{OR}=2.33,95$ \% CI: 1.72 3.17, p<0.001; fig. 4 and fig. 5).

There is no statistical heterogeneity between studies $\left(\mathrm{I}^{2}=0 \%, \mathrm{p}=0.86\right)$, so the fixed effect model was used. The incidence of permanent hypoparathyroidism after TT was equivalent to that of ST and the difference was not statistically significant $(\mathrm{OR}=2.94,95 \% \mathrm{CI}$ : 0.48 18.11, $\mathrm{p}=0.24$; fig. 6)

TABLE 1: GENERAL INFORMATION OF EXPERIMENTAL AND CONTROL GROUPS INCLUDED IN ELIGIBLE STUDIES

\begin{tabular}{|c|c|c|c|c|c|}
\hline $\begin{array}{l}\text { Author and } \\
\text { publication year }\end{array}$ & Group & Number of cases & $\begin{array}{c}\text { Gender } \\
\text { (Male/Female) }\end{array}$ & $\begin{array}{c}\text { Pathological } \\
\text { diagnosis } \\
\text { (NG/TA/TC/Other) }\end{array}$ & $\begin{array}{l}\text { Operation time } \\
\qquad(x \pm s, \min )\end{array}$ \\
\hline Wu & Test group & 20 & $17 / 3$ & $19 / 1 / 0 / 0$ & $95.0 \pm 27.0$ \\
\hline 2004 & Control group & 20 & $1 \mathrm{~h} / 4$ & $1 . / 2 / 0 / 0$ & $104.0 \pm 14.7$ \\
\hline Lombar & Test group & 20 & $10 / 0$ & $5 / 0 / 5 / 0$ & $93.0 \pm 10.6$ \\
\hline 2005 & Control group & 20 & $8 / 2$ & $7 / 0 / 3 / 0$ & $80.5 \pm 22.3$ \\
\hline Peng & Test group & 20 & $24 / 1$ & $19 / 1 / 0 / 0$ & $109.20 \pm 15.24$ \\
\hline 2006 & Control group & 20 & $21 / 4$ & $2 / 17 / 0 / 1$ & $80.67 \pm 15.45$ \\
\hline Wang & Test group & 20 & $20 / 0$ & $1 / 16 / 5$ & $96.00 \pm 26.13$ \\
\hline 2007 & Control group & 20 & $20 / 0$ & $15 / 5 / 0 / 0$ & $95.6 \pm 37.6$ \\
\hline Jiang & Test group & 20 & $19 / 1$ & $17 / 3 / 0 / 0$ & $75.2 \pm 35.0$ \\
\hline 2009 & Control group & 20 & $14 / 6$ & $24 / 1 / 0 / 0$ & $95.83 \pm 23.02$ \\
\hline Yu & Test group & 20 & $19 / 1$ & $\ldots \ldots$ & $110.45 \pm 19.25$ \\
\hline 2010 & Control group & 20 & $19 / 1$ & $2 / 17 / 0 / 1$ & $80.67 \pm 15.45$ \\
\hline $\mathrm{Lu}$ & Test group & 20 & $16 / 9$ & ....... & $96.00 \pm 26 \_13$ \\
\hline 2010 & Control group & 20 & $15 / 7$ & $\ldots \ldots$ & $95.6 \pm 37.6$ \\
\hline Shi & Test group & 20 & $17 / 1$ & $\ldots \ldots \ldots$ & 75. $2 \pm 35.0$ \\
\hline 2010 & Control group & 20 & $13 / 5$ & ....... & $95.83 \pm 23.02$ \\
\hline
\end{tabular}




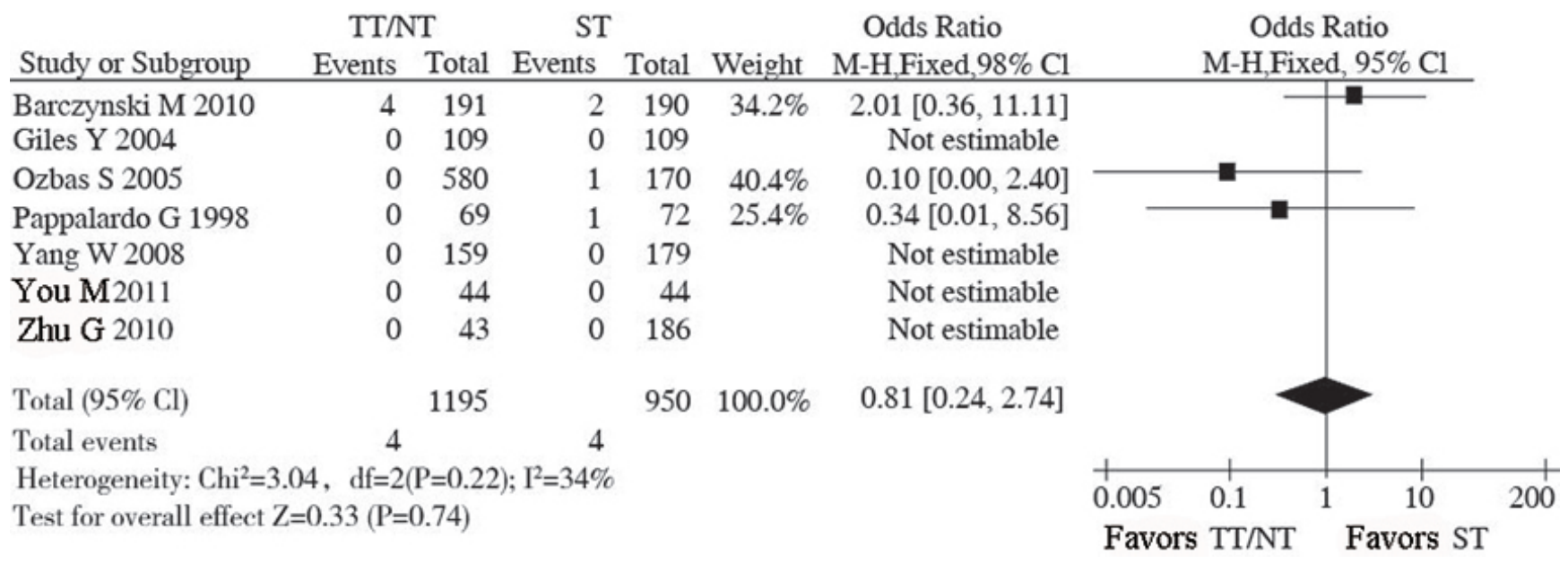

Fig. 1: Meta-analysis of permanent recurrent laryngeal nerve palsy

Study or Subgroup

Barczynski M 2010

Giles Y 2004

Ozbas S 2005

Pappalardo G 1998

Yang W 2008

You M2011

Zhu G 2010

Total $(95 \% \mathrm{Cl})$

Total events

Heterogeneity:

Test for overall effect $\mathrm{Z}=5.43(\mathrm{P}<0.00001)$
TT/NT

ST Events Total Events

$\begin{array}{rrrrr}21 & 191 & 4 & 190 & 14.3 \% \\ 2 & 109 & 1 & 109 & 1.8 \% \\ 117 & 580 & 14 & 170 & 46.2 \% \\ 24 & 69 & 13 & 72 & 16.7 \% \\ 11 & 159 & 9 & 179 & 11.5 \% \\ 6 & 44 & 5 & 44 & 5.9 \% \\ 4 & 43 & 6 & 186 & 3.6 \%\end{array}$

1195

$950 \quad 100.0 \%$
Peto Odds Ratio

Peto, Fixed, 95\% Cl

$4.25[1.89,9.55]$

$1.96[0.20,19.04]$

$2.29[1.46,3.59]$

$2.36[1.12,4.98]$

$1.40[0.57,3.46]$

$1.23[0.35,4.31]$

$4.26[0.84,21.50]$

$2.33[1.72,3.17]$

Fig. 2: Meta-analysis of transient hypoparathyroidism

TT/NT ST

Study or Subgroup Events Total Events

Barczynski M 2010

119

Tota

Peto Odds Ratio

Peto Odds Ratio

Giles Y 2004

$0 \quad 109$

10190

$21.5 \%$

Peto, Fixed, 95\% Cl

Peto, Fixed, 95\% Cl

Ozbas S 2005

1580

$0 \quad 109$

$15.1 \%$

7.35 [0.15, 370.45]

Pappalardo G 1998

$2 \quad 69$

$0 \quad 170$

$15.1 \%$

Not estimable

Yang W 2008

$0 \quad 159$

$63.5 \%$

You M2011

$0 \quad 44$

$1 \quad 72$

$0 \quad 179$

Zhu G 2010

$0 \quad 43$

044

Total $(95 \% \mathrm{Cl})$

1195

$950 \quad 100.0 \%$

Total events

4

4

Heterogeneity: $\mathrm{Chi}^{2}=0.31, \mathrm{df}=2(\mathrm{P}=0.86) ; 1^{2}=0 \%$

$3.64[0.03,393.25]$

$2.05[0.21,20.08]$

Not estimable

Not estimable

Not estimable

Test for overall effect $\mathrm{Z}=1.17(\mathrm{P}<0.24)$

Fig. 3: Meta-analysis of permanent hypoparathyroidism 
www.ijpsonline.com

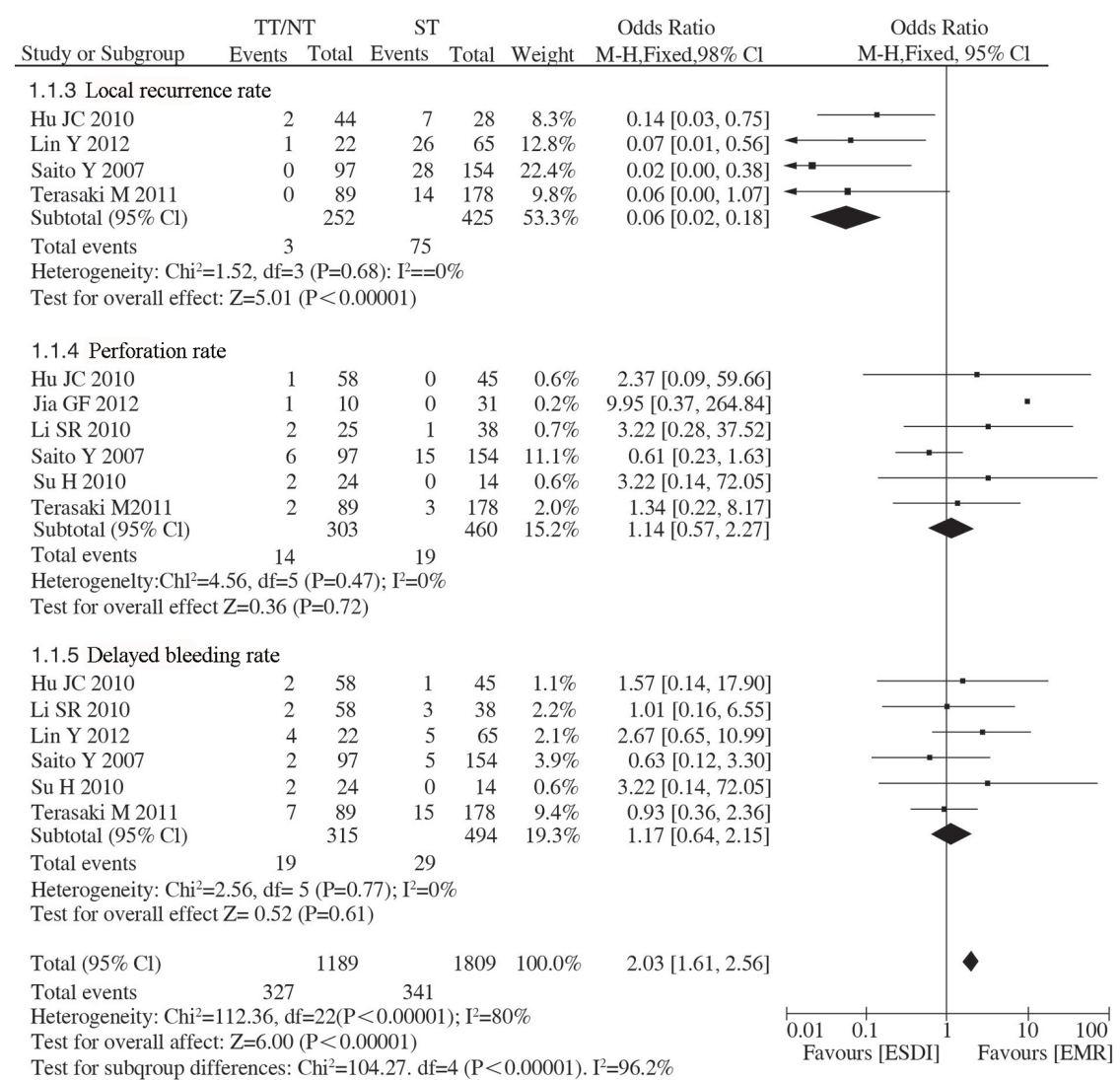

Fig. 4: Meta-analysis of safety and effectiveness

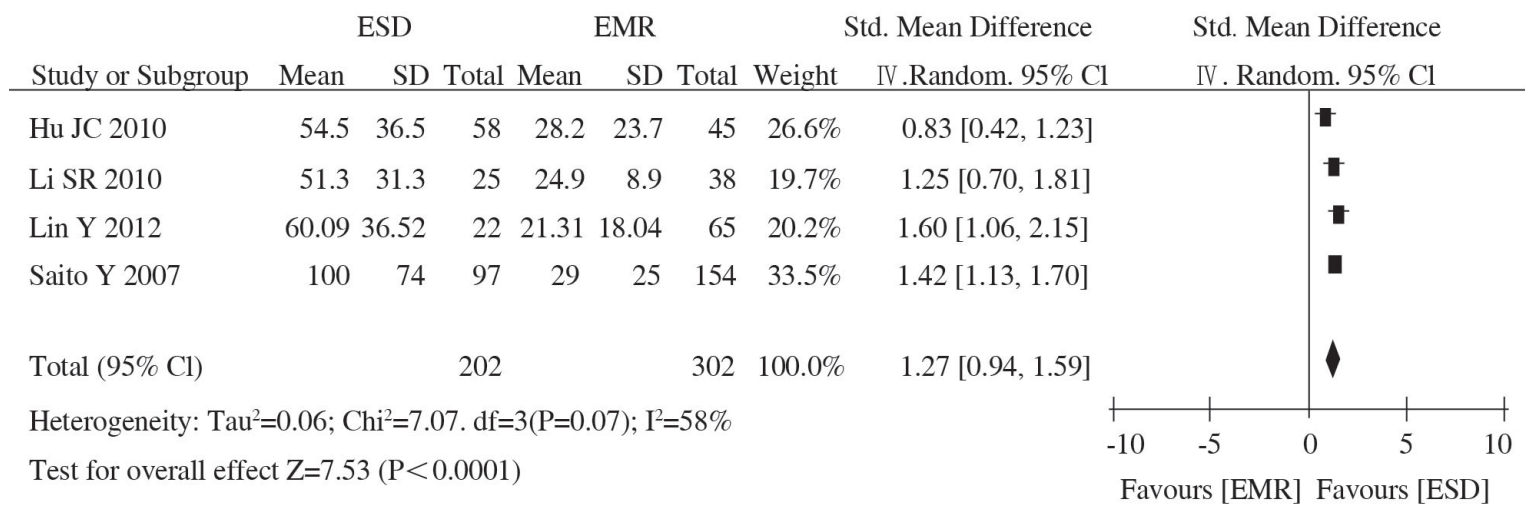

Fig. 5: Meta-analysis of operation time

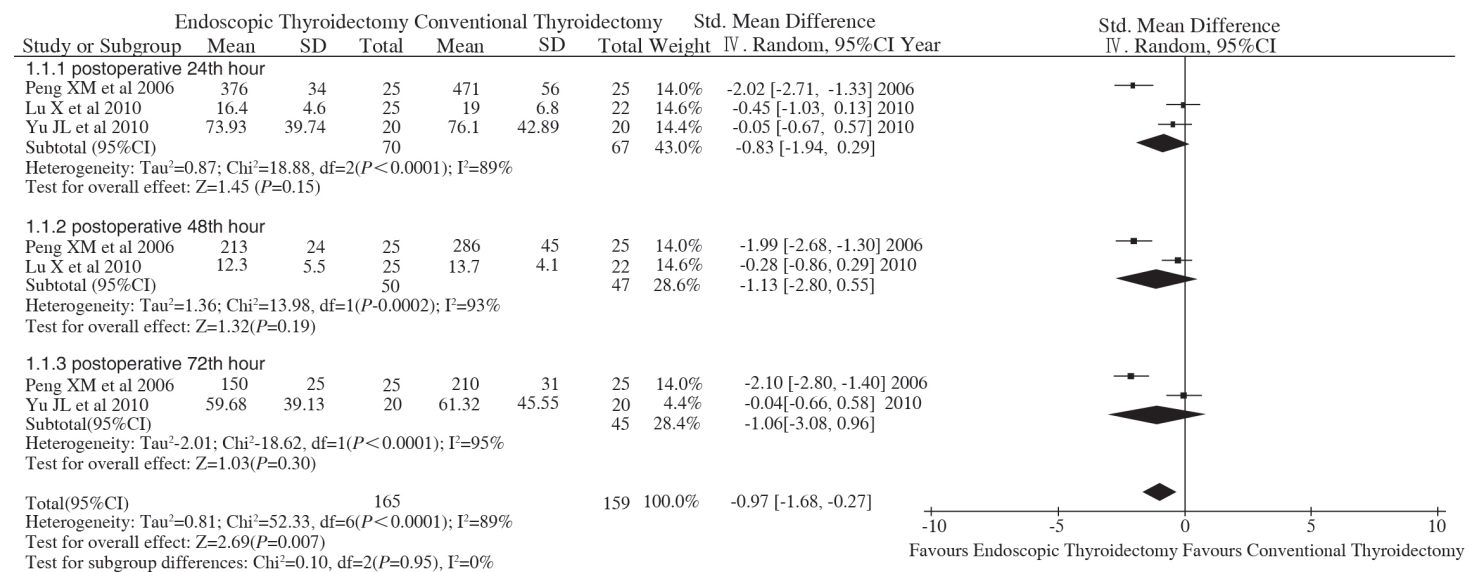

Fig. 6: Meta-analysis of IL-6 level (pg/ml) 
Traditional open thyroid surgery is the main treatment for thyroid surgical diseases. This surgical method is safe and effective, but it will leave obvious surgical scars on the front of the neck and affect the appearance. With the increasing maturity of endoscopic technology and the continuous improvement of surgical instruments ${ }^{[4]}$, various surgical approaches of endoscopic thyroidectomy have been rapidly developed and widely used worldwide ${ }^{[5]}$. However, whether endoscopic thyroidectomy is a minimally invasive operation and whether it is worthy of clinical promotion is still controversial. Some clinical studies have shown that although endoscopic thyroidectomy has good cosmetic effects, its long operation path, wide separation range and long operation time can cause patients to have greater stress response and surgical trauma, which is more traditional than traditional open thyroidectomy has an inhibitory effect on the immune status of the body and is more traumatic. It is only a cosmetic surgery and non-minimally invasive surgery ${ }^{[6]}$. Some clinical studies have shown that endoscopic thyroid surgery is compared with traditional surgery. The cosmetic effect is good. Although it does not meet the minimally invasive standard, it does not increase the trauma to the body. It is safe and feasible ${ }^{[7]}$. There are also clinical studies that show that endoscopic thyroidectomy is different from conventional thyroidectomy. Compared with surgery, the immunosuppressive effect of traumatic stress caused by endoscopic surgery is smaller, which is more conducive to the recovery of the patient's early immune function after surgery ${ }^{[8]}$. Although the pathophysiological mechanism of traumatic suppression of the immune system has not been fully elucidated, experimental and clinical studies have shown that surgical trauma can induce a stress response in the body and surgical trauma is the source of stress ${ }^{[9]}$. The degree of stress response after the body undergoes surgery, is positively correlated with the size of surgical trauma and the levels of peripheral blood cytokines and immune cells after surgery have a good correlation with the degree of surgical trauma ${ }^{[10]}$. In this study, the changes in perioperative cytokines (Interleukin-6 (IL6 ), tumor necrosis factor-alpha (TNF- $\alpha$ ) concentration) and $\mathrm{T}$ lymphocyte subsets (cluster of differentiation $3\left(\mathrm{CD}^{+}\right)$, cluster of differentiation $4\left(\mathrm{CD}^{+}\right), \mathrm{CD}^{+} /$ $\mathrm{CD}^{+}$) were used to reflect the degree of surgical trauma during the perioperative period. To compare and evaluate the differences in the effects of endoscopic thyroid surgery and open thyroid surgery on the body's cellular immune function

Whether endoscopic thyroid surgery is suitable for patients with thyroid cancer is still inconclusive. It is well accepted that ${ }^{[11]}$ for patients with early papillary thyroid carcinoma and follicular carcinoma, no extracapsular invasion or no lymph node metastasis was found in preoperative examination and intraoperative exploration and endoscopic lobectomy is feasible surgery or subtotal resection, preventive central area lymph node dissection should be determined according to the endoscopic technique mastered by the surgeon. Resection of malignant tumors is not recommended unless the carotid sheath can be cleared ${ }^{[12]}$. In clinical practice, it is difficult to make an accurate diagnosis before surgery in many cases and it is usually confirmed as thyroid cancer during or after surgery. Preoperative examinations usually use ultrasound, computed tomography (CT), radionuclide scanning and puncture cytology to diagnose benign lesions as an indication for endoscopic surgery. It is generally believed that the diagnosis of papillary carcinoma by puncture cytology is more difficult. If the cell examination has follicular mutations, rapid intraoperative pathological examination can improve the diagnosis rate ${ }^{[13]}$. There were 2 cases in this group. This situation that the diagnosis was confirmed after surgery is inevitable in clinical practice ${ }^{[14]}$. Recent reports suggest that endoscopic methods are suitable for papillary thyroid carcinoma. The final conclusion remains to be the result of long-term follow-up of a large sample. The main advantage of endoscopic thyroid surgery compared with conventional surgery is that it has good beauty and results, leaving only small scars and no scars on the neck. The success of a large number of endoscopic thyroid surgery proves that the technology is safe and feasible ${ }^{[15]}$. As for the postoperative recurrent laryngeal nerve, superior laryngeal nerve and parathyroid tissue damage, which is generally worried in advance, from the current reports, the incidence is no different from traditional surgery, or even lower ${ }^{[16]}$. This is due to the magnification of the endoscope, so that the surgeon can clearly identify the superior laryngeal nerve, recurrent laryngeal nerve and parathyroid glands, so that they can be protected. The postoperative pain of the anterior chest, underarm approach and conventional surgery was basically the same and the underarm approach had the best cosmetic effect ${ }^{[17,18]}$. Conventional surgery leads to complications such as paresthesia and swallowing discomfort. This group of cases shows that with the accumulation of experience of the surgeon and the application of ultrasonic scalpel, the bleeding during the operation and the operation time can be greatly reduced. In a retrospective study of 427 cases, 
the outcomes of this method were not significantly different from those of traditional surgery in terms of operation time and postoperative complications ${ }^{[19]}$. The postoperative aesthetic effect has been confirmed. The results through the questionnaire are the same. The short postoperative hospital stay and short recovery time make patients feel that endoscopic thyroid surgery is more minimally invasive than traditional surgery. In general, small-incision endoscopic thyroid surgery has been successful and its applications will increase in the future. In the process of developing this technology, surgeons should pursue minimally invasive endoscopic thyroid surgery, including reducing trauma and shortening the operation time. Compared with traditional surgery, it has complicated surgical operations, uses high-tech equipment and high medical costs. The surgeon is required not only to have rich experience in traditional thyroid surgery, but also proficient endoscopic surgery skills. More importantly, the development and promotion of neck endoscopic surgery also requires a change in the concept of surgeons, i.e. accepting minimally invasive surgery is the future direction of development.

At present, the World Health Organization has begun to use evidence-based medicine to formulate a list of essential medicines and basic medical measures; Australia formulates treatment guidelines in the field of surgery based on evidence-based medicine every year. Provide the basis for medical decision-making; the British Health Technology Coordination and Evaluation Center is responsible for the overall plan of the British health technology evaluation and guides the quality and direction of national health research. Evidence-based medicine is influencing the medical practice, medical education and clinical research of these countries, prompting them to complete the transition from empirical medicine to evidence-based medicine. The significance of the implementation of evidence-based medicine in my country is mainly as follows. The Ministry of Health has learned from the principles and methods of evidence-based medicine and established a health technology access management office that is conducive to scientific health decision-making in my country and promulgated health technology access management measures. Implement access management for important health technologies that are expensive, influential and controversial. The State Administration of Traditional Chinese Medicine, the State Drug Administration and the National Family Planning Commission are also actively learning and introducing evidence-based medicine, exploring the use of it to improve the scientific nature of the national drug policy and family planning policy and to promote the modernization of traditional Chinese medicine. Meanwhile, evidence-based medicine is also of great practical and historical significance for helping to establish and improve the standardized national health resource database and to realize the real-time collection, in-depth mining and secondary development of basic data. Evidence-based medicine, with its scientific attitude of speaking with evidence, continuous updating and after-effect evaluation, provides managers with clear management ideas and methods, uses evidence to guide practice and monitors the development, production, evaluation and adverse reactions of new drugs. Especially it has extremely high reference value for promoting the modernization of Chinese medicine, going abroad and creating brands. Moreover, China has a vast territory, obvious regional differences in medical services, unbalanced allocation of health resources, different diseases in different regions and differences in the quality and level of medical workers. Popularizing knowledge of evidence-based medicine can improve the quality of medical services by continuously updating and improving the clinical knowledge and professional skills of doctors on the premise of basically not increasing medical expenses, which will ultimately benefit the government and the public.

Gagner completed the world's first endoscopic parathyroidectomy. In 1997, Hiiseher et al. took the lead in completing endoscopic thyroid lobectomy and succeeded, endoscopic thyroid surgery has developed rapidly worldwide. Subsequently, the surgical path of endoscopic thyroidectomy has also been continuously updated. At present, the main clinical paths are transsternal approach, subclavian approach, subaxillary approach and breast approach. Endoscopic thyroid surgery utilizes the features of the endoscopic magnification system and remote operation to shrink or hide the surgical incision and the cosmetic effect is beyond doubt. A large number of studies have shown that, in addition to cosmetic effects, endoscopic thyroid surgery has advantages over open thyroid surgery in terms of postoperative complications and recovery. However, some scholars questioned that endoscopic surgery has a long path, a wide range of separation and a long operation time, which may increase the trauma to the body. Due to the lack of multi-center large-scale prospective randomized controlled studies, whether endoscopic thyroid surgery is truly minimally invasive is still widely controversial. This study uses the principles and methods of evidence-based medicine 
and strictly follows the requirements of Cochrane systematic review to comprehensively search and screen RCTs concerning $\mathrm{CD}^{+} \mathrm{T}$ cells, $\mathrm{CD} 4^{+} \mathrm{T}$ cells, $\mathrm{CD}^{+} / \mathrm{CD}^{+}$changes in the perioperative period, objective to evaluate the impact of the two surgical methods on the immune status of the body at different times during the perioperative period, to explore whether endoscopic thyroid surgery is minimally invasive compared with open thyroid surgery, to guide clinical measures to avoid excessive stress response or adjustment imbalance, reduce the harm caused by stress to the body, so that patients can obtain the dual effects of beauty and minimally invasiveness and provide more reliable evidence for clinical practice. IL-6 is the body stimulated by lymphocytes under surgical trauma. It is secreted by monocytes, macrophages and endothelial cells. Its biological effect is mainly to induce the production of $\mathrm{T}$ lymphocytes and $\mathrm{B}$ lymphocyte antibodies. It is a sensitive marker and mediator that reflect the early stage of tissue damage. IL- 6 can act on various target cells in the body are not only important markers of tissue damage, but also play an important role in the body's acute and dangerous phase response after injury. IL-6 is also an important inflammatory mediator that mediates surgical trauma and stress. Surgical trauma can directly or indirectly cause the synthesis and release of IL-6 to increase the level of IL6 , which is associated with surgical stimulation, tissue damage and postoperative complications. Occurrence is closely related. The level of IL-6 rises 12 to $48 \mathrm{~h}$ earlier than the onset of complications. The detection of serum IL-6 levels before and after surgery can help determine the degree of surgical stress and predict the occurrence of complications. It can be seen from the included research data that the levels of IL-6 in peripheral blood of patients in the experimental group and the control group increased significantly after surgery, and serum IL-6 reached a peak at $24 \mathrm{~h}$ after surgery. In this study, meta-analysis showed that there was no statistical difference between the experimental group and the control group in the peripheral blood IL-6 concentration at $24 \mathrm{~h}(\mathrm{p}=0.15), 48 \mathrm{~h}(\mathrm{p}=0.19)$ and $72 \mathrm{~h}(\mathrm{p}=0.30)$ after surgery. Therefore, it can be considered that compared with traditional open thyroid surgery, endoscopic thyroid surgery has no difference in the impact of early surgical trauma on the immune function of the body. This meta-analysis study shows that ESD surgery time is significantly longer than EMR. Analysis of the reason may be related to the delicate and complex operation of ESD and the proficiency of different surgeons on ESD can also affect the operation time. The operation time of ESD is significantly longer than that of EMR, which may pose potential risks for elderly patients and patients with cardiopulmonary insufficiency. Therefore, ESD operations should be performed by experienced physicians. Real-time monitoring of the patient's vital signs during the operation. Proficiency in ESD techniques is an important condition for reducing complications. Our meta-analysis has the following limitations: the literature included in this meta-analysis is not an RCT and some studies have poor baseline comparability between the ESD treatment group and the EMR treatment group. This is related to the endoscopic detection rate of colorectal LST. The detection rate of colorectal endoscopy at home and abroad is relatively low and it is difficult to implement multicenter largesample RCTs or prospective trials. Although the included studies are all retrospective analyses, the quality of the original studies is fair after Newcastle-Ottawa Scale (NOS) quality evaluation; Only part of the literature provides data on tumor mass resection rate, complete tumor resection rate, local recurrence rate, operation time, perforation rate and delayed bleeding rate, which may affect the reliability of the conclusion; There is a large heterogeneity in the pooled analysis of operation time, but the combined effect size of individual studies excluded by sensitivity analysis is still statistically significant and the direction of the forest plot results has not changed; the included studies are mainly from Asia (Japan and China). Western countries are not included and most of them are single-center, small-sample studies. The results of this pooled analysis may not be applicable to all patients worldwide.

This is related to the difference in the endoscopic detection rate of colorectal LST between the east and the west. TNF- $\alpha$ is a cytokine produced by activated monocyte-macrophage system cells and has a wide range of activities. Surgical trauma stress can directly or indirectly cause the increase of TNF- $\alpha$ synthesis and release, enhance phagocytosis cause hyperplasia oxide is released in large quantities and the main mechanism of its increase is related to excessive stimulation of the body by monocytes and macrophages. Produced in large quantities and enters the bloodstream can cause systemic reactions, such as being an endogenous pyrogen to cause fever in the body; acting on monocytes and vascular endothelial cells, stimulating the secretion of IL-6 and IL-6; acting on liver cells, increasing acute phase protein synthesis in the liver, etc.; it can also directly stimulate the hypothalamus thermoregulation center to cause fever. The production of TNF- $\alpha$ in the early posttraumatic period can be used as the main effector of 
tissue damage and cell-mediated immunopathological response and is an important indicator of response to surgical trauma and acute stress. This research passed meta. The analysis showed that the peripheral blood TNF- $\alpha$ of the test group and the control group. There was no statistically significant difference in concentration at $24 \mathrm{~h}$ after surgery. Therefore, it can be considered that compared with traditional open thyroid surgery, endoscopic thyroid surgery has no difference in the impact of surgical trauma and acute stress on the immune function of the body. Intraoperative perforation and delayed bleeding are common complications of endoscopic resection and treatment of colorectal tumors. Therefore, it is necessary to avoid perforation and bleeding during the operation. This meta-analysis study showed that the intraoperative perforation rate and delayed bleeding rate in the ESD treatment group were slightly higher than EMR, but there was no statistical difference. Tanaka et al. reported that the rate of surgery related perforation in ESD resection of 70 colorectal tumor lesions was as high as $10 \%$, but the improvement of ESD endoscopic treatment equipment can greatly reduce the occurrence of perforation. Although most of the perforations can be sutured with endoscopic titanium clips, only a few larger perforation foci need to be treated surgically. Endoscopists still need to continuously improve endoscopic treatment equipment and improve endoscopy.

The treatment technique reduces the perforation rate. This meta-analysis study shows that ESD surgery time is significantly longer than EMR. Analyzing the reason may be related to the delicate and complex operation of ESD and the proficiency of ESD by different surgeons can also affect the operation time. The operation time of ESD is significantly longer than that of EMR and it may have potential risks for elderly patients and patients with cardiopulmonary insufficiency. Therefore, ESD operations should be performed by experienced physicians. Real-time monitoring of the patient's vital signs during the operation. Proficiency in ESD techniques is an important condition for reducing complications. Our meta-analysis has the following limitations: The literature included in this metaanalysis is not an RCT and some studies have poor baseline comparability between the ESD treatment group and the EMR treatment group. This is related to the endoscopic detection rate of colorectal LST. The detection rate of colorectal endoscopy at home and abroad is relatively low and it is difficult to implement multi-center large-sample RCTs or prospective trials. Although the included studies are all retrospective analyses, the quality of the original studies is acceptable after NOS quality evaluation; Only part of the literature provides data on tumor mass resection rate, complete tumor resection rate, local recurrence rate, operation time, perforation rate and delayed bleeding rate, which may affect the reliability of the conclusion; There is a large heterogeneity in the aggregate analysis of the operation time, but the combined effect size of the individual studies excluded by the sensitivity analysis is still statistically significant and the direction of the forest plot results has not changed.

The included studies are mainly from Asia (Japan and China). Western countries are not included and most of them are single center, small sample studies. The results of this pooled analysis may not be applicable to all patients worldwide. This is related to the difference in the endoscopic detection rate of colorectal LST between the East and the West. In conclusion, the results of this meta-analysis show that ESD has significant advantages over EMR in terms of en bloc resection rate, complete resection rate and local recurrence rate does not increase surgery related complications. For laterally growing large intestine tumors with a diameter $>2 \mathrm{~cm}$, ESD may be preferred. In view of the small sample size of some of the original studies included in this meta-analysis, which may affect the accuracy of the conclusions, it is still necessary to design a multicenter, large sample, high quality, long-term followup randomized controlled study strictly in accordance with the Consolidated standards of reporting trials (CONSORT) standard for further demonstration.

Complications of endoscopic thyroid surgery and prevention of transthoracic approach during endoscopic thyroid surgery, it is necessary to establish the anterior chest wall space and perfuse $\mathrm{CO}_{2}$ to maintain the operating space, which may cause subcutaneous emphysema, mediastinal emphysema, hypercapnia and skin deficiency. Blood, necrosis, etc., of course, may also produce the same bleeding as traditional surgery; damage the superior laryngeal nerve, recurrent laryngeal nerve and parathyroid glands, resulting in complications such as vocal cord paralysis, swallowing paresthesia and hypocalcemia after surgery. During the initial domestic endoscope thyroid surgery, rare complications such as damage to the trachea and neck blood vessels occurred. However, the $\mathrm{CO}_{2}$ pressure during endoscopic surgery is maintained at $6 \mathrm{mmHg}$ and the subcutaneous emphysema will naturally subside after the operation. We have carried out endoscopic thyroid surgery so far, there has been no case of hypercapnia and mediastinal 
emphysema. During the operation, the ultrasonic knife is used to coagulate the blood vessels and the cutting thyroid tissue has a significant hemostatic effect. As long as the operation is performed properly, bleeding complications can be avoided. When performing a total thyroidectomy, carefully identify the parathyroid glands and protect the nutrient blood vessels of the parathyroid glands to avoid hypocalcaemia after surgery. The incidence of postoperative recurrent laryngeal nerve palsy is about $2.2 \% \sim 3.4 \%$, which often occurs in the early stage of this operation, lack of experience and is related to the larger mass. Generally, it can heal by itself after a follow-up of about 2 mo. The reason for the analysis may be the reversible damage to the nerve caused by the heat conduction of the ultrasonic knife. Therefore, it is necessary to close the proper thyroid capsule when coagulating blood vessels and cutting tissues and expose the recurrent laryngeal nerve as much as possible during total thyroidectomy and subtotal resection. Be protected; the separation and cutting off the back of the thyroid tissue should be combined with blunt separation to maintain a clear surgical field, minimize the use time of the ultrasonic knife and avoid heat damage to the nerve. There are reports that the incidence of recurrent laryngeal nerve injury in endoscopic thyroid surgery is similar to that of traditional surgical methods. Therefore, we have reason to believe that with the accumulation of experience and the improvement of technology, the incidence of complications of endoscopic thyroid surgery can be reduced to the traditional level of surgery.

Endoscopic surgery and microsurgery are two surgical methods for the treatment of thyroid diseases. Thyroid surgery is the most important parameter for comparing these two surgical methods. However, which surgical method has a higher therapeutic effect is still controversial. In 2009, Tabaee et al. conducted Meta results showing that endoscopic surgery has a higher complete tumor resection rate than traditional microsurgery. The reason may be that the endoscope can observe and remove the tumor from different angles, avoiding the blind angle of the operation field under the microscope and improving the total tumor resection rate. In 2012, de Klotz et al. conducted meta and found that the incidence of bleeding after endoscopic surgery was lower than that of microsurgery. The results of this study showed that compared with microsurgery, the incidence of bleeding after endoscopic surgery was significantly reduced $(\mathrm{p}<0.05)$ and the number of hospital stays was significantly reduced $(\mathrm{p}<0.05)$; and the total tumor resection rate, operation time and postoperative period were significantly reduced in the two groups. There was no significant difference in the incidence of cerebrospinal fluid leakage, brain/meningitis, postoperative diabetes insipidus and postoperative hormone recovery $(p>0.05)$. The low incidence of bleeding after endoscopic surgery and the few days of hospitalization may be attributed to: The length of the endoscope and the small cross-section are suitable for operation in narrow cavities and channels; the light source of the endoscope reaches a deeper surgical field. The degree of clarity is significantly better than that of an operating microscope; The endoscope tube itself can have a side view and the tumor and normal pituitary tissue can be clearly distinguished during the operation; after most of the tumor is removed, the thyroid sidewall can be clearly displayed and can stop bleeding under direct vision.

Intraoperative damage to important structures can be avoided and serious postoperative complications can be reduced. Therefore, the intraoperative field of vision is clear, the injury is small, which helps reduce bleeding, there are fewer postoperative complications, postoperative recovery is quicker and the hospital stay is shortened. This study has certain limitations. For example, most of the included literature is a retrospective study and the randomization and blinding of some literatures are unclear. The included cases in each group may be biased and the research quality is not high; different literature comes from different sources. In research centers, the experience and proficiency of surgeons are not exactly the same, which may be another important source of bias. Incorporating rigorously designed high-quality clinical randomized controlled studies for systematic reviews is an effective way to solve the above problems.

\section{Conflicts of interest:}

The authors declared no conflicts of interest.

\section{REFERENCES}

1. $\mathrm{Bu} \mathrm{N}$, Cai H, Qiu F. Risk analysis and nursing care of endoscopic thyroidectomy through oral vestibular approach. Mod Clin Nursing 2018;17(12):21-4.

2. Xu J. Application progress of endoscopic thyroidectomy under complete oral route. Minim Invas Med 2016;11(03):378-80.

3. Luo X, Liu X, Xu N. The effect of endoscopic single-port and double-areola thyroid surgery on the ipsilateral axillary path. Chin J Gen Surg 2016;10(02):156-8.

4. Huang X, Liang F, Yu S. Endoscopic thyroid papillary adenocarcinoma surgery. J Otolaryngol Ophthalmol Shandong Univ 2016;30(02):13-6.

5. Zhou T, Chen K, Ai L. Nursing analysis of endoscopic subtotal thyroidectomy. Chin J Prev Treat Endem Dis 2015;30(06):549. 
6. Lian D, Zhang N, Peng J, Zhu B, Gong K, Amin B. Endoscopic unilateral thyroidectomy under different approaches. Chin $\mathrm{J}$ Gen Surg 2015;30(9):695-7.

7. Qin Z, Li F. Progress in clinical application of needle endoscopic thyroidectomy. Minim Invas Med 2014;9(4):45962.

8. Tao Z, Wei Q, Yang Y. 62 cases of thyroid surgery with small anterior neck incision suspension without gas injection. Zhejiang Pract Med 2014;19(3):198-221.

9. Cai R. Evaluation of the efficacy and safety of thyroidectomy in patients of different ages. China Med Guide 2014;16(6):966-9.

10. Fan Q, Gong K, Zhu B, Zhang N. Clinical experience in the treatment of retrosternal goiter under complete endoscopic surgery. Peking Univ J Med 2014;46(3):488-91.

11. Wang Z, Pan Z, Rao S. Early experience and complication analysis of complete areola approach endoscopic thyroidectomy. Zhejiang Medical Association Surgery Branch. 2013 Zhejiang Surgery Academic Annual Conference Paper Collection. Zhejiang Sci Technol Assoc 2013;1.

12. Zhang Z, Sun Y, Xu W, Yang Y. Analysis of 62 cases of thyroid surgery with small anterior neck incision suspension without air injection. Zhejiang Provincial Medical Association Surgery Branch. 2012 Zhejiang Surgery Academic Annual Conference Proceedings. Zhejiang Sci Technol Assoc 2012;1.

13. Wu S, Fan Y. Experience of total endoscopic thyroidectomy through a single incision under the axilla. Chin J Gen Surg 2012;2:160-1.

14. Li J, Ma J. Application experience of stripping method in modified Miccoli operation. Chin J Modern Surg 2011;15(05):330-2.
15. Li X. Surgical cooperation and nursing care of 87 cases of endoscopic thyroidectomy. Creating a patient safety culture-the 15th National Operating Room Nursing Academic Exchange Conference of the Chinese Nursing Association (Volume 1). Chin Nurs Assoc 2011;3.

16. Kunus S. Clinical efficacy and analysis of endoscopic and open partial thyroidectomy in the treatment of benign thyroid diseases. Xinjiang Med Univ 2011.

17. Li S, Zhang H, Wei Y, Wu Y, Zhang Q, Qian J, et al. Practical neck endoscopic minimally invasive thyroid surgery. J Clin Exp Med 2010;9(23): 1763-1765.

18. Liang F, Hong Y, Huang X, Zheng Y, Cai Q, Sun W. Clinical analysis of 292 cases of thyroid surgery with small anterior neck incision. Chin J Otorhinolaryngol Head Neck Surg 2010;10:861-3.

19. Wang W, Fang Q, Li J, Jin P, Li J, Li Z. Discussion on the efficacy of endoscopic thyroid surgery. China Higher Med Educ 2010;8:134-5.

This is an open access article distributed under the terms of the Creative Commons Attribution-NonCommercial-ShareAlike 3.0 License, which allows others to remix, tweak, and build upon the work non-commercially, as long as the author is credited and the new creations are licensed under the identical terms

This article was originally published in a special issue,

"Therapeutic Perspectives in Biomedical Research and Pharmaceutical Sciences and their Nursing Methods"

Indian J Pharm Sci 2021:83(4)Spl issue "66-76" 